\title{
PERCEPCIÓN DE SOLEDAD EN LA MUJER
}

Recibido: Enero de 2011 - Revisado: Febrero de 2012 - Aceptado: 30 de Marzo de 2012

\author{
Por: Paula Cristina Rios Zapata, ${ }^{1}$ Nora Helena Londoño Arredondo ${ }^{2}$
}

\section{RESUMEN:}

El propósito del presente estudio fue establecer el estado de la cuestión de las investigaciones sobre la soledad y la percepción de la misma. La soledad, en sí misma es considerada como factor de riesgo para trastornos del estado de ánimo, consumo de drogas y suicidio, entre otros, pero también como opción de vida que expresa cambios socioculturales de la época contemporánea. La transformación del rol de la mujer en la pareja, la familia, y la sociedad, conlleva modificación de conceptos sobre el fenómeno de la soledad; para comprenderle no solo, desde una perspectiva negativa, sino también como opción de vida en la mujer. Se concluye a partir de la revisión de antecedentes investigativos, la necesidad de estudiarla junto con otras variables como la autoestima, el apoyo social y la personalidad, las cuales pueden determinarle como riesgo o como factor protector.

\section{PALABRAS CLAVE:}

soledad, percepción de soledad, apoyo social, autoestima personalidad, soledad en la mujer.

\begin{abstract}
:
The purpose of this study was to establish the status of the issue of investigations into loneliness and its perception. Loneliness itself is considered as a risk factor for mood disorders, drug consumption, and suicide, among some others, but also as a life choice which expresses social and cultural changes of the contemporary era. The transformation of the role of the woman in the couple, the family, and the society, require the modification of concepts about the phenomenon of loneliness; in order to understand not only from a negative perspective, but also as an option of life in women. It is concluded from the review of investigative background, the need to study it together with other variables such as selfesteem, social support, and personality, which can determine it either as a risk or a protective factor.
\end{abstract}

\section{KEY WORDS:}

Loneliness, Perception of Loneliness, Social Support, Self-Esteem, Personality, Loneliness in Women.

\footnotetext{
${ }^{1}$ Psicóloga. Docente/investigadora Universidad Católica de Oriente Antioquia - Colombia. E-mail: prios@uco.edu.co. Sector 3 Cra. $46 \mathrm{~N}^{\circ}$ 40B-50, Rionegro, Antioquia, Colombia

${ }^{2}$ Magister en Psicología. Docente/investigadora Universidad de San Buenaventura Medellín Colombia. E-mail: nora.londono@usbmed.edu.co;nora_londono@yahoo.es
} 


\section{Introducción.}

La soledad se ha entendido como un estado subjetivo que se relaciona con la condición de aislamiento físico (Peplau y Perlman, 1982). Surge como una respuesta ante la falta de una relación particular e implica un desequilibrio en el nivel deseado y logrado de interacción socioafectiva, cuyas consecuencias pueden afrontarse de manera negativa o positiva, dependiendo del grado de control que ejerce el individuo sobre su déficit relacional (Montero, López y Sánchez, 2001).

El concepto de soledad se ha diferenciado de otros conceptos como el apoyo social y aislamiento, a su vez, se ha ligado a cuadros de depresión, intento de suicidio y consumo de alcohol, haciendo de ésta, un factor a tener en cuenta en la salud mental de una comunidad (Montero et al. 2001; Expósito y Moya, 1999; Gaviria, González, Palacio, García y López, 2009).

Algunas investigaciones han encontrado asociación entre la percepción de soledad y variables como apoyo social (Expósito et al., 1999; Cobb, 1976), autoestima (Expósito et al., 1999; Castelló, 2005) y personalidad (Fierro y Cardenal, 2001; Hojat, 1982; Cheng y Furnham, 2002), sin que se encuentren estudios específicos relacionados con la mujer.

La soledad puede ser concebida como una condición negativa para quienes se perciben solos o se sienten aislados o excluidos de la red de apoyo social, como una experiencia negativa que sobreviene a la persona cuando su red de relaciones sociales es deficiente, bien sea por su tamaño o por su calidad. De esta manera, se le describe en términos de la discrepancia entre los niveles de contacto social deseado y los contactos reales mantenidos (Peplau y Perlman, 1982).

En los últimos años, se ha incrementado el número de personas que viven solas y que constituyen una nueva categoría social: singulares (singles), solos, solteros, solitarios urbanos cuya soledad tiene más que ver con la elección de un tipo de vida que con la antigua soltería (Alborch, 2001).

Con relación al apoyo social, estudios como los de Expósito y Moya (1999), han concluido que la soledad tiene relación con aspectos muy particulares de las relaciones (y del apoyo social), concretamente con aquellos que son más subjetivos, emocionales, relacionales, globales y profundos. La soledad no es simplemente la falta de relaciones sociales (o de apoyo social), sino que se trata de una experiencia que sobreviene a la persona cuando ésta percibe la ausencia de un tipo muy específico de relación, estrechamente vinculado a la persona, o fuente que hace posible esa relación o suministra el apoyo social.

En muchos estudios se evidencia la relevancia que tiene el apoyo social para la salud en general de las personas, con aspectos como conexiones sociales, soledad, red de apoyo, vínculos familiares, nivel de ingresos, estrategias de socialización y relación parental entre otros, dan cuenta de ello (Chen y Chung, 2007; Wiseman, Mayseless y Sharabony, 2006).

La autoestima ha sido asociada a los estudios de la soledad, como lo reporta el estudio de Expósito y Moya (1999), donde señalan la existencia de tres fuentes de las que procede la autoestima y que interesan por la importancia que otorgan al aspecto valorativo e interpersonal y por lo tanto, por su relación con la experiencia con la soledad y son: a) 
reflejarse en el otro, b) comparación social, y c) evaluación de aspectos colectivos o sociales. Estos autores plantean la relación inversa entre la soledad y la autoestima. De este modo, cuanto mayor es el sentimiento de autoestima menos probable es que se sientan solas a lo largo de su vida (Peplau y Perlman, 1982; Borys y Perlman, 1985).

Por otro lado, la soledad ha sido un factor asociado a la salud mental y a ciertas características de personalidad. Estos aspectos podrian incidir directamente en la percepción negativa o positiva de la soledad, o de las fuentes de apoyo. En esta dirección, algunos autores han estudiado la personalidad asociada a la soledad y a la calidad de vida (Cheng y Furnham, 2002; Hojat, 1982).

Montero, López y Sánchez (2001), investigaron el fenómeno de la soledad, con el propósito de conocer algunos de los componentes psicológicos vinculados con la experiencia de ésta, a través de un análisis metaanalítico. Realizarón una revisión de la bibliografia científica vinculada con este fenómeno, destacando tres perspectivas conceptuales: a) la filosófica, b) la social-antropológica y c) la psicológica; y describiendo seis esquemas conceptuales que intentan explicar en la soledad identificando sus alcances y limitaciones, tanto teóricas como metodológicas. Estos esquemas se agrupan en dos clasificaciones:

1) Esquemas fenomenológicos: estos esquemas hacen referencias a las características cualitativas de la experiencia solitaria (Sadler y Johnson, 1980; Rokach, 1988).

2) Esquemas cognitivos: esquemas que se relacionan con los procesos de percepción social e interpersonal (Jong-Gierveld y Raadschelder, 1982; Peplau y Perlman, 1982; Weiss, 1973, 1982). Y finalmente se propuso un esquema integrativo que evalúa la experiencia de soledad en términos de frecuencia.

Si bien, tradicionalmente en la literatura sobre el tema se ha establecido un paralelismo entre soledad y apoyo social, en tanto que ambos conceptos hacen referencia a las relaciones sociales que mantienen las personas, algunas investigaciones como las realizadas por Expósito y Moya (1999), han puesto de manifiesto que la soledad, a pesar de estar relacionada con determinados aspectos del apoyo social, es un fenómeno diferente que hace referencia a aspectos más subjetivos, emocionales, profundos y globales y que por tanto, tendrá efectos más devastadores sobre la vida de las personas que la falta de apoyo social. En esta investigación se presentarón datos de 786 personas de ambos sexos y diferentes edades, nivel educativo, estado civil, etc., que contestarón un cuestionario que incluía la tercera versión de la Loneliness Scale, de la Universidad de California, Los Ángeles (UCLA, Loneliness Scale; Russell, Peplau y Cutrona, 1980), la medida de Apoyo Social de Dunkel-Schetter, Feinstein y Herbert (1986), así como medidas de autoestima y calidad de vida.

Los resultados obtenidos apoyarón sus predicciones acerca de la especificidad de la experiencia de soledad, concluyendo que la soledad es un fenómeno relativo a las relaciones sociales, pero no en el sentido cuantitativo que tradicionalmente se ha entendido. La soledad tiene que ver más con aspectos muy específicos de las relaciones (y del apoyo social), concretamente con aquellos que son más subjetivos, emocionales, relacionales, globales y profundos.

Un elemento común dentro de los conceptos de soledad es la percepción de soledad, o soledad percibida, que se puede describir y definir mejor que el concepto mismo de ésta por su nivel de 
abstracción. Si bien en general el concepto ha sido considerado como la ausencia de compañía (soledad social), algunos autores han utilizado el término para referirse a una experiencia subjetiva mucho más compleja que tiene su origen en el modo en el que el individuo percibe la calidad de sus relaciones (soledad emocional) (Russell et al, 1978; 1980; 1984; Weiss, 1973; 1982). Algunos autores consideran la soledad como un rasgo, mientras que otros la conciben como un estado pasajero.

\section{La edad y la cultura ante el fenómeno de la soledad.}

Oviedo y Cortés (2007), realizarón una aproximación cualitativa sobre las representaciones sociales de la soledad a partir de un acercamiento a los saberes, los sentimientos y las experiencias que poseen los niños, los adolescentes, los adultos y los ancianos. Dentro de esta investigación se asumió la perspectiva de realidades múltiples, con diferencias entre ellas que no pueden resolverse a través de procesos relacionales o aumentando los tamaños muestrales.

Rokach y Neto (2005), examinarón la influencia de la edad y la cultura sobre las causas percibidas de la soledad. 1.346 canadienses y portugueses de todos los sectores sociales se ofrecierón para responder a un cuestionario de 82 ítems, de respuesta sí / no, para reflexionar sobre las causas de su soledad. El cuestionario utilizado en este estudio estaba compuesto por factores que describen las causas de la soledad: dificultades personales, déficit de desarrollo, relaciones íntimas insatisfactorias, reubicación / separaciones importantes, y la marginalidad social. Las diferencias de género entre y dentro de los grupos también fueron examinados. Se compararon 4 grupos de edad: adolescentes (13-18 años), adultos jóvenes (19-30), adultos (31-58) y personas mayores (60 y mayores). Los resultados indicaron que las causas de la soledad se perciben de manera diferente dependiendo de la edad y la cultura. Con relación a la cultura, se distinguieron dos estilos de organización humana; una comunidad orgánica, parecida a la sociedad de Portugal, y una sociedad atomizada, semejantes a la sociedad americana y canadiense. En la comunidad orgánica, el conjunto es la base de la sociedad y la persona no podría vivir sin ella, los individuos se basan en gran medida en la comunidad para su apoyo, poseen sentido de pertenencia; ésta sociedad hace mucho énfasis en los lazos cercanos de la familia; por el contrario las culturas americana y canadiense hacen énfasis en el logro individual y en las relaciones de competencia, en éstas culturas son más difíciles las alianzas con otros. Acá es más común una disminución de los contactos de grupo, con los vecinos, y se enfatiza la autonomía y el éxito personal. Con relación a la edad encontrarón que los adolescentes no atribuyen su soledad a sus propias características de personalidad, tanto como los jóvenes, adultos y ancianos. Los adultos jóvenes y los mayores pueden ser conscientes de sus defectos personales y saben que estos les pueden impedir alcanzar sus objetivos. En su mayoría se separan de sus familias durante los años de juventud y construyen amistades con otros adultos, si por el contrario no lo logran, pueden terminar en una percepción de inutilidad al sentirse no aceptados socialmente y rechazados, y más al ver a otros adultos socialmente exitosos.

\section{Causas de la soledad.}

Rokach (2005), investigó acerca de las causas de la soledad en jóvenes sin hogar, en América del Norte. Los participantes fueron seleccionados al azar en las escuelas secundarias, la comunidad local y una universidad local. Los resultados identificaron 5 causas de la soledad: características de personalidad inadecuadas, déficit de desarrollo, relaciones íntimas 
insatisfactorias, reubicación o separaciones importantes y la marginalidad social. Los resultados indicarón que las causas de soledad en los jóvenes sin hogar son significativamente diferentes de las de la población en general.

Rokach, Lechcier-kimiel y Safarov (2006), estudiarón la soledad de las personas con discapacidad fisica, la cual tiene un efecto profundo en la propia calidad de vida, relaciones sociales y bienestar emocional. Se ha encontrado que la soledad es una compañera frecuente de los afectados con enfermedades crónicas que resultan en discapacidades físicas. Este estudio examinó los aspectos cualitativos de esa soledad. Participarón 593 voluntarios. Las personas con discapacidades físicas se compararón a la población sin discapacidad (en general), y luego se dividieron en cinco subgrupos homogéneos (es decir, personas con esclerosis múltiple, osteoporosis, parkinson, artritis, y "otros") que se compararon entre sí y con la muestra de población general que estaban sanos y no enfermos crónicos. Los resultados indican que la soledad de aquellos con discapacidades físicas es significativamente más alta que la de la población en general.

\section{Soledad y salud mental.}

Gaviria et al. (2009), identificarón, en una muestra de población antioqueña con trastorno depresivo mayor y antecedente de conducta suicida, las características que se asocian a intentos de suicidio de alta letalidad; mediante la evaluación de 140 sujetos con diagnóstico de trastorno depresivo mayor y antecedente de, al menos, un intento de suicidio, por medio de una entrevista diagnóstica semiestructurada. De acuerdo con las características del intento de suicidio más serio, los sujetos se dividieron en grupos de menor letalidad (letalidad leve y moderada) y mayor letalidad (letalidad alta y extrema), los cuales se compararon en variables socio demográficas y clínicas. Encontrarón que los sujetos con intentos de suicidio de letalidad alta y extrema con mayor frecuencia vivian solos, tenían antecedente de más de un intento de suicidio y presentaban dependencia a nicotina y a sustancias psicoactivas diferentes al alcohol. En el análisis multivariado se estableció que vivir solo y la dependencia a sustancias psicoactivas se asociaron de manera independiente con los intentos de suicidios de letalidad alta y extrema.

\section{Perspectivas frente a la conceptualización de la soledad.}

Entre las perspectivas conceptuales que han abordado el estudio de la soledad se destacan la filosófica, la social-antropológica y la psicológica (Montero et al. 2001).

Perspectiva filosófica: Desde el punto de vista filosófico, la soledad es entendida como una condición inescapable en la búsqueda de la autoconciencia. Está arraigada en la realidad primaria del individuo, inmanente y subjetiva (Montero et al. 2001).

Perspectiva socio-antropológica: En contraste con la perspectiva filosófica, la aproximación social - antropológica está representada por estudios antropológicos y culturales que analizan las manifestaciones de la soledad en diversas actividades artísticas, tales como la literatura, la poesía, la pintura, la música, entre otras. Puede encontrase conceptualizaciones de la soledad, donde ésta es vista como un proceso de retroalimentación adaptativo, utilizado por el individuo para informarse del nivel de estimulación e interacción social, al que está expuesto, en términos de cantidad y de forma (Montero et al. 2001). Peplau y Perlman (1982) identificaron "tres características comunes de la soledad: a) es el resultado 
de deficiencias en relaciones interpersonales; b) es una experiencia subjetiva que contrasta con la evidencia física del aislamiento social; y c) es una experiencia estresante y displacentera" (Montero et al. 2001, p.20). Estas características se derivan de la naturaleza de los déficits interpersonales, que pueden darse por carencias afectivas o por carencias cognoscitivas. Las carencias afectivas, hacen referencia a una necesidad de intimidad y/o apego. Las carencias cognoscitivas, hacen referencia a una percepción de estimulación social por debajo del nivel óptimo considerado por el individuo.

Perspectivas psicológicas: desde la perspectiva psicológica, existen diversas definiciones para la soledad, tal vez, la más antigua sea la de Sullivan quien la concibió como una experiencia displacentera, asociada con la carencia de intimidad interpersonal. Continuando con esta línea, Young $(1982,1990)$ concibió la soledad como la ausencia real o percibida de relaciones sociales satisfactorias, que pueden ser acompañadas por manifestaciones de "distress" psicológico. Para Weiss $(1973,1982)$, quien fuera llamado "el padre de la investigación sobre la soledad", esta es una respuesta ante la ausencia de una provisión de relación particular. Otros autores la definen como una discrepancia entre las relaciones interpersonales actuales y las deseadas. Para Peplau y Perlman (1982) "la soledad es una experiencia displacentera que ocurre cuando la red de relaciones sociales de una persona es deficiente en algún sentido importante, ya sea cualitativa o cuantitativamente" (p.21).

El concepto de soledad, puede agruparse en dos esquemas conceptuales básicos, el fenomenológico y el cognoscitivo:

El esquema fenomenológico, agrupa varios postulados conceptuales, que conciben la soledad como "una forma de autoconciencia, y parten de la idea, que el ser humano tiene como necesidad básica el afecto, mientras que la soledad es el resultado de la insatisfacción de este requerimiento básico" (Montero et al. 2001, p.21).

Como principales representantes de este esquema, se encuentran: Sadler y Johnson (1980), con un modelo de cuatro dimensiones de la soledad (Fig.1); y Rokach (1988), quien propuso un Modelo Multidimensional de Antecedentes de la Soledad (Fig.2).

Figura 1 . Dimensiones de la soledad: Sadler y Johnson (1980)

\begin{tabular}{|c|c|}
\hline $\begin{array}{c}\text { CÓSMICA } \\
\text { Vinculada con tres } \\
\text { percepciones del yo, en relación } \\
\text { con la Naturaleza, con Dios y } \\
\text { con el propósito de vida. }\end{array}$ & $\begin{array}{c}\text { SOCIAL } \\
\text { Relacionada con la } \\
\text { percepción de falta de relación al } \\
\text { interior de pequeños grupos (ej. } \\
\text { Asilados, jubilados, exiliados) }\end{array}$ \\
\hline $\begin{array}{c}\text { CULTURAL } \\
\text { Asociada con la } \\
\text { percepción de disfunción social } \\
\text { basada en conflictos de valores, } \\
\text { normas, creencias y actitudes. }\end{array}$ & $\begin{array}{c}\text { INTERPERSONAL } \\
\text { Referida a la autoconciencia } \\
\text { de déficits en las interacciones } \\
\text { con personas significativas para } \\
\text { el sujeto. }\end{array}$ \\
\hline
\end{tabular}

Retomado de Montero, et al., 2001 
Figura 2. Rokach (1988). Modelo Multidimensional de antecedentes de la soledad.

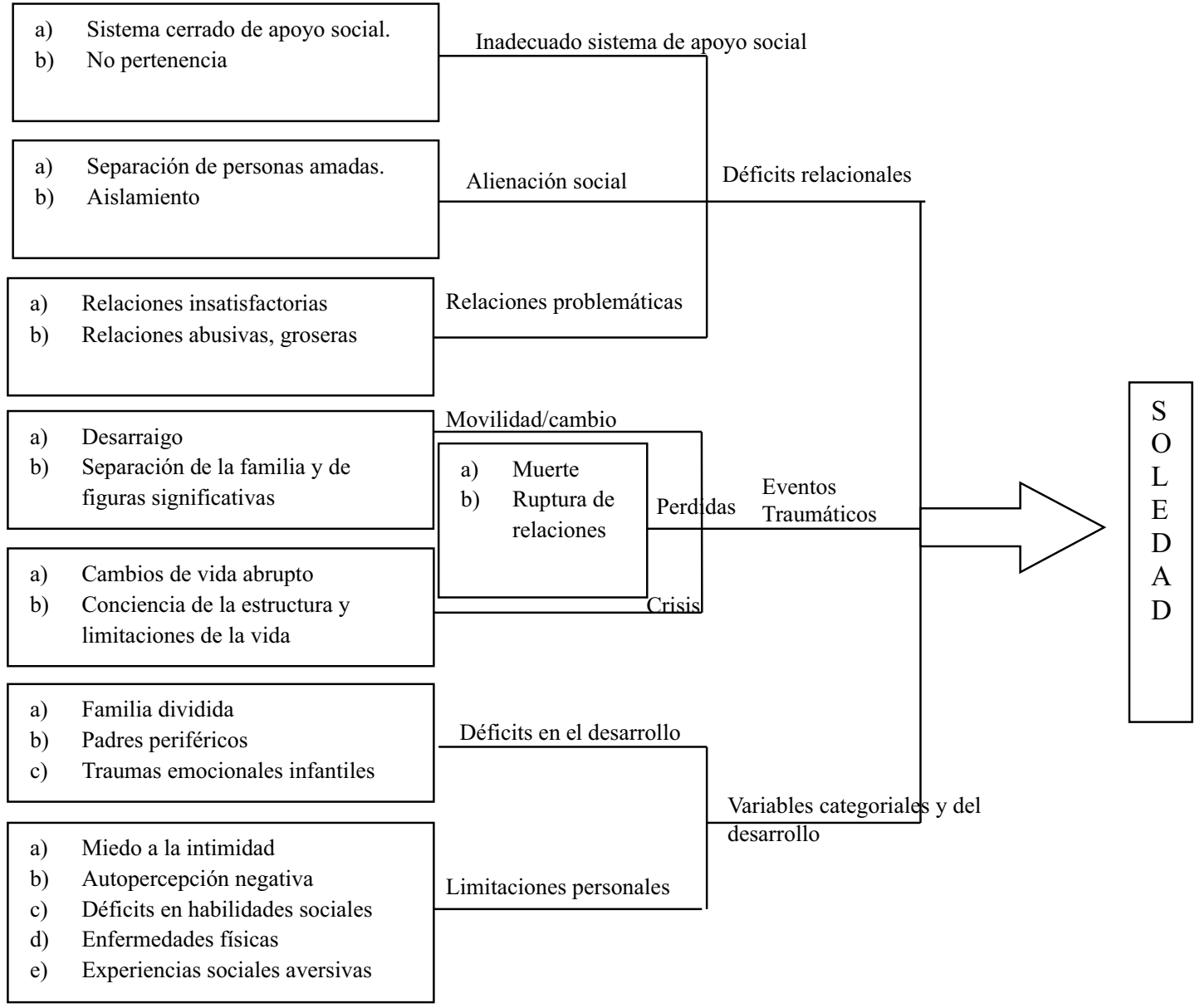

Retomado de Montero, et al., 2001

El esquema cognoscitivo, está caracterizado por el postulado fundamental, que es la evaluación subjetiva que hace el sujeto de la situación interpersonal en la que se encuentra. Está evaluación es multideterminada, por algunos factores que pueden predecir, con mayor precisión en magnitud y frecuencia, la experiencia de soledad (Montero et al. 2001). En este esquema, se concibe la soledad como el resultado de dos tipos de déficits, uno asociado con la carencia de una red de apoyo social y otro, vinculado con la falta de una figura de apego particular. Ambos tipos de soledad son provocados por "disrupciones en el sistema de apego" (Paloutzian y Janigian. En: M. Hojat y R. Crandall, 1989). Como principales representantes de este esquema se encuentran: Weiss, quien hace énfasis en el déficit relacional, para él (Fig.3):

La soledad de aislamiento emocional ocurre ante la ausencia de un apego emocional cercano y solamente puede remediarse por la incorporación de otro apego emocional o por la 
reintegración del que se había perdido; en contraste, la soledad de aislamiento social está vinculada con la ausencia de una red social y ésta sólo puede remediarse por el acceso a dicha red. (Montero et al. 2001 p.22).

Figura 3 . Weiss (1973). Esquema bidireccional de la soledad

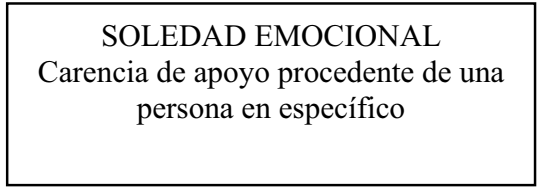

Retomado de Montero, et al., 2001
SOLEDAD SOCIAL

Carencia de apoyo procedente de un grupo de referencia

Peplau y Perlman (1982), indicarón que cada persona tiene un nivel óptimo de interacción social. Cuando las relaciones sociales de un sujeto son subóptimas, éste experimenta soledad; en contraste; cuando el sujeto se enfrenta a un contacto social excesivo, puede experimentar "hacinamiento" o sentir "una invasión de privacía". Las evaluaciones de las relaciones sociales de un sujeto están influidas por las comparaciones con la experiencia pasada del mismo sujeto y con las experiencias de otras personas (Fig. 4) (Montero et al. 2001, p.22).

Figura 4 . Peplau y Perlman (1982). Esquema de disonancia cognitiva

Cercanía e intimidad deseada

Retomado de Montero, et al., 2001

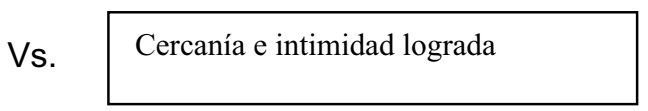

Jong-Gierveld (1987), pone énfasis en los procesos cognoscitivos que median entre las características de la red social y la soledad. Define la soledad como una experiencia personal y subjetiva, y propone un Modelo Multidimensional de Soledad, donde hace énfasis en la "importancia de las percepciones e interpretaciones personales de la red de relaciones sociales" (Montero et al. 2001, p.23). Este modelo considera cuatro factores:

1) las características descriptivas de la red social,

2) las evaluaciones subjetivas de la red social,

3) las variables sociodemográficas, $y$

4) las características de la personalidad (Fig.5). 
Figura 5 . Jong-Gierveld (1987). Modelo multidimensional de la soledad.

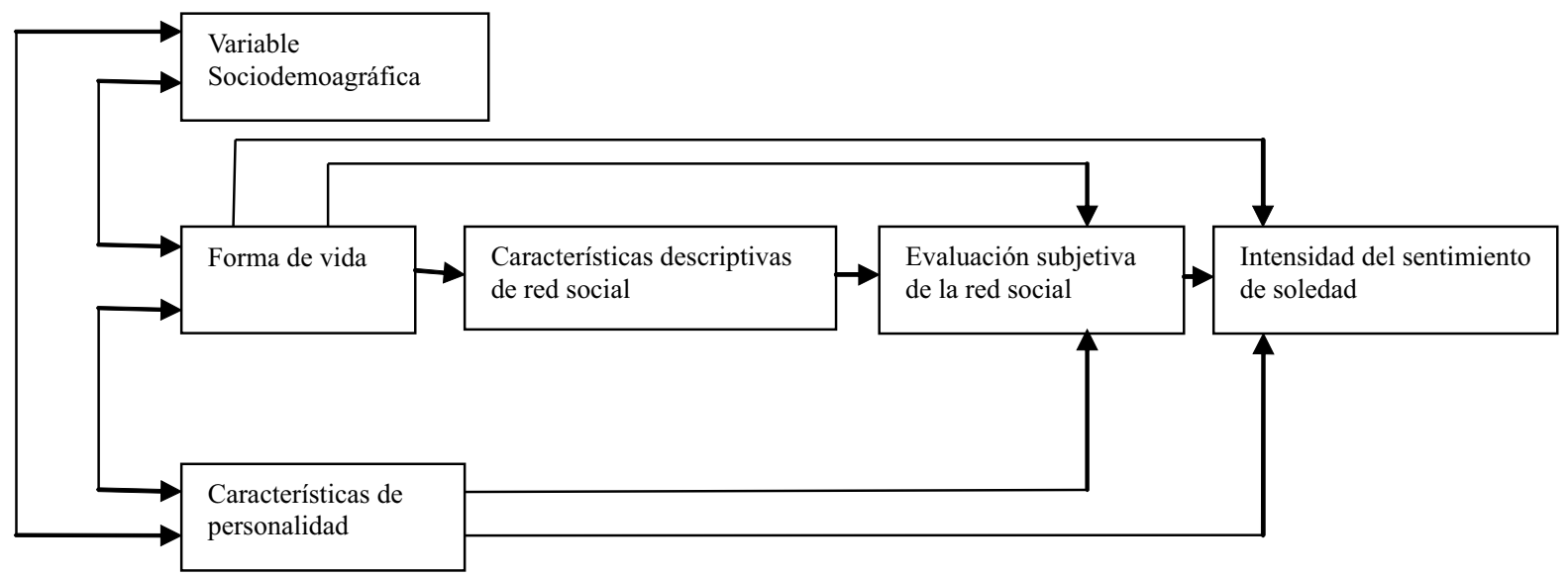

Retomado de Montero, et al., 2001

Jong- Gierveld y Raadschelders (1982), consideran tres dimensiones de la soledad: a) Evaluación por parte del sujeto de la situación social donde se encuentra, b) el tipo de déficit social que experimenta y c) la perspectiva temporal asociada con la soledad (Montero et al. 2001, p.24).

Young (1982), identificó tres tipos de soledad: a) crónica, de larga duración, donde la persona se siente incapaz de establecer relaciones sociales satisfactorias; b) situacional, vinculada usualmente con situaciones estresantes como el divorcio o la muerte de la pareja, los padres o los hijos; y c) transitoria, es la más común y se asocia con brotes breves del sentimiento de soledad (Montero et al. 2001).

\section{Mujer y soledad}

Mujeres solas han existido siempre, en tiempos y lugares distintos, y seguramente nunca han formado parte del núcleo mayoritario de la sociedad. La sola existencia de las mujeres solas desafiaba o alteraba tanto la dinámica tradicional hombre - mujer, como las funciones esenciales del matrimonio.

Diferentes escritoras como Jules Michelet, y J. Simon, se referian a la mujer sola en estos términos: "La mujer perece, si no tiene hogar ni protección. Si hay algo que la naturaleza nos enseña, de manera evidente, es que la mujer está hecha para ser protegida, para vivir de muchacha junto a su madre y de esposa bajo la tutela y autoridad de su marido" y "las mujeres están hechas para mantener oculta su vida; fuera del hogar y del matrimonio no hay salvación", según Rousseau, "la mujer, sólo lo es en relación al hombre" (Alborch, 1999 p. 30). A través de la historia nos hemos encontrado con varios modelos de mujer sola: En el siglo XVIII: Las mujeres solteras, vivian en casas de familia, trabajando como empleadas domésticas. En la época victoriana, se le ofrecian a las mujeres dos alternativas extremas: madres o prostitutas, la solterona era entonces la imagen de pureza y sacrificio. Durante este 
siglo, se originó además la Revolución Industrial en Inglaterra, hacia 1780, aproximadamente, que se extendió por Francia, Alemania, Estados Unidos y Rusia a lo largo de los siglos XIX y XX, y trajo muchos cambios sociales, entre ellos, el ingreso de la mujer a la fuerza laboral (Uribe, 2008). Produjo, como indica M. Perrot, una redefinición que determinaba lo público y lo privado, así como los roles sexuales, sin embargo se estableció una equivalencia entre lo público para lo masculino, lo privado para lo femenino. Y al mismo tiempo, que se proclamaba la igualdad y los derechos de los individuos, se les negaban a las mujeres, y eran excluidas de la vida pública, quedando confinadas a lo privado. La mujer sola suscitaba suspicacia, reprobación o burla.

En el siglos XIX, las mujeres prefirieron trabajar como obreras en las fabricas a trabajar en el servicio domestico. Otras consiguieron trabajos de más liderazgo como: misioneras, enfermeras, maestras. El teatro, la opera y el ballet también abrió las puertas a las mujeres solas. Pero por esta incursión en el mundo laboral, muchas mujeres fueron atacadas y discriminadas por su cambio de rol y fuerón marginadas por médicos y sexólogos que las estigmatizaron como "lesbianas". La defensa de su identidad sexual las llevo a desafiar esta tendencia, y constituyeron en el siglo XIX los primeros movimientos feministas organizados. A finales del siglo XIX, éstas mujeres emancipadas fueron viajeras célebres, que afirmaron con esto, su libertad personal. Ya en el siglo XX, se producen grandes avances y uno de los acontecimientos más relevantes fue la presencia de las mujeres como protagonistas de la vida social, resultado, del movimiento de mujeres. En ese siglo, como consecuencia del crecimiento de las aglomeraciones urbanas y la industrialización, las familias se desintegraron, liberando así, una mano de obra valiosa para el desarrollo económico de la nueva sociedad política.

La sociedad se convierte en el horizonte de los solteros y al mismo tiempo, atrae y fabrica solitarios. Las grandes ciudades constituyen auténticas reservas de mujeres solas, resultado de la emigración del campo a la ciudad. Su participación en el proceso industrial supuso una gran revolución que cambió su suerte, e inició para ella, una nueva era, en la que fue definitivo el acceso a la educación (Alborch, 1999).

En la actualidad, se puede decir que casarse ya no es un fin en sí mismo. Tanto los hombres como las mujeres pueden sobrevivir, social y económicamente, sin matrimonio: pero no obstante, los movimientos feministas del siglo pasado y el cambio de estereotipos sociales y culturales, para la mujer sigue siendo un punto crítico el estar sola, ya sea porque su soledad sea su elección, cosa que no será bien vista en su entorno cultural, o porque sea una soledad impuesta por las circunstancias de vida.

En el estudio de Alborch (2001), se reportaron algunas ideas encontradas en la población en general, que son prejuicios sobre la mujer sola tales como: "las mujeres solteras son unas histéricas que se derrumban bajo una profunda crisis de confianza"; "las mujeres sin hijos están deprimidas y desorientadas" y "las mujeres solteras son más numerosas cada día”.

Lo cierto es que nos encontramos ante un problema, no solo conceptual sino terminológico, dada la multiplicidad de significados que encierra la palabra "soledad"; desde la institucional (relacionada con el estado civil) a la íntima (Alborch. 2001).

Y esta confusión más los estereotipos culturales hacen que la soledad sea vista y en algunos casos sentida, como un "problema", como es sabido la sociedad impone roles de manera que 
los individuos van desarrollando una serie de actitudes, sentimientos y comportamientos, de acuerdo a sus funciones (la maternidad en el caso de las mujeres, la guerra y la competitividad en el caso de los hombres). La personalidad masculina y femenina se define de acuerdo con los valores y comportamientos asignados según el género al que pertenecen. A su vez estos valores y comportamientos asignados dan lugar a una serie de estereotipos, es decir, opinión ya hecha que se impone a los individuos de una comunidad y que incide en las actitudes y expectativas que tenemos frente a las mujeres y los hombres, atribuyéndoles determinados rasgos de personalidad que no son innatos, sino adquiridos.

Estos estereotipos hacen que muchas mujeres en condición de soledad, se perciban a sî mismas en una situación de exclusión o aislamiento, debido a la fuerte presión cultural y social que se ejerce sobre ellas por su soledad o por no tener una relación de pareja estable. Asociado a esto, se encuentran factores como la autoafirmación, autoestima, autonomía, los estilos de afrontamiento, las habilidades sociales, la personalidad, entre otros, que hacen que una mujer asimile el estar sola a un castigo o a un fracaso y que la independencia y libertad pueda ser percibido por muchas como sinónimos de soledad y exclusión.

Todo esto puede ir en aumento o detrimento de la salud mental de la mujer que se encuentra en condición de soledad. En investigaciones, se ha encontrado en mujeres, relación entre la soledad con características de las interacciones que mantiene en su medio social, de la misma manera que se ha encontrado en ellas, una relación entre soledad y la intimidad percibida de las relaciones, deficiencias en la autorevelación o la ausencia de un confidente (Expósito et al, 1999).

\section{Soledad y apoyo social.}

La soledad, a pesar de estar relacionada con determinados aspectos del apoyo social, es un fenómeno diferente que hace referencia a aspectos más subjetivos, emocionales, profundos y globales, que tendrá efectos más devastadores sobre la vida de las personas que la falta de apoyo social (Expósito, et al., 1999).

La soledad es un fenómeno relativo a las relaciones sociales, pero no en el sentido cuantitativo que tradicionalmente se ha entendido. La soledad no es simplemente la falta de relaciones sociales (o de apoyo social), sino que se trata de una experiencia que sobreviene a la persona cuando ésta percibe la ausencia de un tipo muy específico de relación y que además está estrechamente vinculado a la persona o fuente que hace posible esa relación o que suministra el apoyo social.

En ocasiones, la soledad puede ser entendida como una experiencia desagradable que se produce cuando la red social de una persona es deficiente, de forma significativa en calidad o cantidad, y ello es asociado con sentimientos negativos.

Se entiende por Apoyo Social "la ayuda, consejo, bienestar e información que uno recibe de su red social, incluyendo la familia, amigos, compañeros de trabajo y vecinos" (Cobb, 1976).

En el Apoyo Social, se resalta su carácter multidimensional, se encuentran al menos tres tipos: a) emocional, que hace referencia a la expresión por parte de los demás de gestos o conductas de cariño y estima; b) informativo, se refiere a consejo y guía; e c) instrumental, se trata de recibir de los demás bienes tangibles y asistencia en determinadas tareas que lo precisen (Martínez y García, 1995). 
El Apoyo Social y la soledad, tienden a confundirse, aunque se encuentran básicamente dos aspectos que los diferencian:

1) El apoyo social, aunque suele darse dentro de las relaciones sociales, no implica relaciones propiamente dichas, sino interacciones en las que ocurren ciertas conductas específicas y esporádicas. La soledad, en cambio, se refiere a las relaciones interpersonales, especialmente cuando son intensas y duraderas.

2) El apoyo social está más relacionado con los aspectos cuantitativos de las relaciones, mientras que la soledad, se relaciona con la dimensión cualitativa, es decir, con la calidad y no la cantidad (Expósito, et al, 1999).

La experiencia de la soledad es mucho más compleja que la ausencia de relaciones sociales o de apoyo social. Como la define Weiss (1973) "la soledad no es simplemente un deseo de compañia, cualquier compañía, sino más bien la ausencia de una relación muy específica" (Expósito, et al, 1999, p. 300).

De acuerdo a Expósito y Moya (1999) la soledad está relacionada con aspectos subjetivos/cualitativos, emocionales, relacionales, globales y profundos, que describen de la siguiente manera:

1) Subjetivos/cualitativos: la soledad refleja una 'una percepción subjetiva del individuo de deficiencias en sus relaciones' (Russell, Cutrona, Rose y Yurko, 1984).

2) Emocionales: la soledad es un estado subjetivo que resulta no tanto del aislamiento físico de los demás, como de la ausencia de dosis suficientes de significatividad (contacto emocional) en las relaciones (Wheeler, Reis, y Nezleck, 1983).

3) Relacionales: “...la soledad parece ser más una cuestión de ausencia/pérdida de conexiones o relaciones íntimas que la ausencia/ pérdida de contacto social en general" (Reis, 1990; Wheeler, et al, 1983)

4) Globales: “...la experiencia de la soledad afecta de manera general la vida de la persona y estará por tanto, relacionada con índices globales de bienestar (como la calidad de vida percibida)".

5) Profundos: La soledad, se trata de un sentimiento profundo y dificilmente apreciable por un observador externo, causado principalmente por la ausencia de personas especiales, con las que se mantienen relaciones íntimas, lo cual impide o dificulta experimentar sentimientos de seguridad, reconocimiento, cuidado, afecto y valía (pp. 300-301).

Algunos autores han considerado el apoyo social percibido, como una variable de gran relevancia en los procesos de salud - enfermedad (Aranda, Pando, y Aldrete, 2002). Y puede ser conceptualizado desde una dimensión estructural (tamaño de la red social) o desde una dimensión funcional (multifactorial) (Gottlieb, 1983). Este implica la confianza en que el apoyo social está disponible si se necesita (Barrón, 1996). 


\section{Soledad y autoestima}

La autoestima es uno de los temas más estudiados de la psicología, debido a esto, se encuentra un sinnúmero de definiciones de ella. Una de las definiciones más comunes es la planteada por Blascovich y Tomaka (1991) para quienes la autoestima es "la extensión en la cual uno se aprecia, valora, aprueba o se gusta a sí mismo" (Expósito et al. 1999, p.301).

Se retoma el concepto, por ser un factor que interfiere en la soledad y en la percepción de soledad. Entendiendo que la soledad está estrechamente ligada a la autoestima. De alguna manera las personas que desarrollan sentimientos positivos hacia sí mismas, se sienten queridas y valoradas por otras personas, se sienten "elegidas" y "especiales" para alguien que de la misma manera es especial para ellas mismas (Expósito y Moya, 1999). Evidenciándose la importancia que tienen los demás, en la conformación y consolidación de la autoestima.

De esta forma la relación que se mantenga con uno mismo se rige por parámetros similares que la relación que se sostiene con los demás. Si se tiene sentimientos positivos hacia los demás, preocupación por ellos, se les protege, se intenta hacerles la vida más agradable y más fácil, y se asegura que no se pierdan sus derechos, se busca lo mejor para ellos, se desea tener contacto con ellos y se disfruta por tanto de su presencia. Lo mismo sucede con nosotros mismos.

Si los sentimientos que nos dirigimos son positivos, es decir, si tenemos una autoestima suficiente y saludable, nos preocupamos por nosotros mismos, nos protegemos, intentamos hacernos la vida más agradable y más fácil, nos aseguramos de que no se violen nuestros derechos, buscamos lo mejor para nosotros mismos y disfrutamos cuando estamos en soledad, sin renunciar evidentemente a nuestro trato con los demás, que, nutre en cierto modo dichos sentimientos positivos hacia nosotros (Castelló, 2005).

Según Castelló (2005), “...la existencia de sentimientos y auténticas relaciones del sujeto consigo mismo, está determinado por su carácter social, por la vinculación afectiva del individuo con los demás. La autoestima vendría a ser un premio, algo 'reforzante' que estaría precedido en circunstancias normales de relaciones positivas con los demás, las cuales se interiorizarían y asumirian. La carencia de autoestima sería el resultado de una relación frustrante y poco igualitaria con el entorno interpersonal, y denotaría el escaso valor adaptativo de esta serie de circunstancias desfavorables para el individuo" (p. 254-255).

En tal sentido, se puede entender como una vinculación afectiva, del individuo, con otros, tiene un carácter adaptativo, y acorde con una perspectiva biológica evolutiva, esto se revierte en una mayor cooperación entre ellos mismos y en una autoestima adecuada de cada uno de ellos, lo que a su vez conduce a una vida más productiva y satisfactoria. Por lo tanto, ésta autoestima, y el sentimiento de satisfacción que vendría determinado por la relación positiva del sujeto consigo mismo, le propiciaría la vinculación afectiva con los demás, creándose un círculo beneficioso en el que existiria satisfacción, cooperación y productividad (Castelló, 2005). Pero por otro lado, la autoestima de las personas vinculadas afectivamente, no va a depender solamente de la relación positiva con los demás, sino que se fortalece además por las valoraciones, juicios y sentimientos, que la persona experimente hacia sí misma.

Algunos autores citan entonces tres fuentes de las que procede la autoestima: a) El reflejarse en el otro: las personas pueden evaluarse a sí mismas, basándose en el conocimiento o en lo 
que imaginan respecto a cómo los demás las evalúan; b) Comparación social: comparación con otros semejantes a nosotros o significativos y c) Evaluación de aspectos colectivos y sociales: parte de nuestra autoestima deriva de los grupos sociales a los que pertenecemos, considerados en su globalidad (Expósito y Moya, 1999).

Otros, hablan de dos fuentes o suministros de autoestima, uno externo y otro interno: a) Externo: proviene del entorno interpersonal, de la interiorización de sentimientos y valoraciones positivas de los demás, y b) Interno: es en el mismo proceso pero realizado por el propio individuo. Y la unión y equilibrio entre estos, conforma la autoestima del individuo. Así "si la autoestima es baja, la relación del individuo consigo mismo será negativa, caracterizada por falta de cariño hacia sí mismo, autodesprecio, autocrítica, etc. Si la autoestima es correcta, la relación del sujeto consigo mismo será buena y existirá amor propio, satisfacción, autoprotección, defensa de los intereses y deseos, etc.” (Castelló, 2005, p. 256).

\section{Soledad y personalidad}

Fierro y Cardenal (2001) en su investigación sobre pertinencia de estilos de personalidad y variables cognitivas como indicadores de salud mental, que prosigue una línea de estudio que han emprendido los autores para explorar correlatos de personalidad y también de variables cognitivas en la personalidad "sana" y "madura".

Frecuentemente, cuando se habla sobre la personalidad de alguien, se hace referencia a lo que diferencia a una persona de los demás, incluso lo que la hace única. A este aspecto de la personalidad se conoce como diferencias individuales. Para algunas teorías, ésta es la cuestión central y prestan una atención considerable a tipos y rasgos de las personas, entre otras características, con los cuales categorizar o comparar... algunas personas son neuróticas, otras no; algunas son más introvertidas, otras más extravertidas, y así sucesivamente.

Pero para entender lo que es realmente "personalidad" se debe remitir al origen de la palabra misma y como ha ido evolucionando su significado con el transcurrir del tiempo, debido a un sinfin de investigaciones que han abordado su naturaleza.

La palabra "personalidad" proviene del término latino "Persona", que significa "la máscara del actor", debido a que una de las características del teatro clásico era el uso de una máscara, que cubría la cara del actor durante toda la obra. Este carácter de fijeza fue lo que llevó a la psicología clásica a utilizar el término de personalidad para definir el carácter permanente del individuo. De hecho, se definió la personalidad como "la función psicológica por la que un individuo se considera como un yo único y permanente". En la psicología actual se define la personalidad como "la organización dinámica de los aspectos intelectuales, afectivos, impulsivos, volitivos, fisiológicos y morfológicos del individuo".

El tópico de la personalidad ha sido tratado por filósofos, médicos, psicólogos que desde la antigüedad se han interesado por su complejidad, encontrando desde, los antiguos griegos a Hipócrates, con su teoría de los cuatro tipos de personalidad: Sanguíneo, flemático, melancólico y colérico, basados en el tipo de humores (fluidos corporales). Teoría que siendo tan antigua tuvo gran influencia en varios teóricos e investigadores modernos como Adler, Pavlov y el mismo Eysenck quien aún siendo conductista y quien consideró que los hábitos aprendidos eran de gran importancia, consideró que nuestras diferencias en las 
personalidades surgen de nuestra herencia, por lo tanto, estuvo principalmente interesado en el temperamento; planteó en su teoría la presencia de tres dimensiones de la personalidad: neuroticismo, extraversión-introversión y psicoticismo.

Pero, el interés y la investigación de la personalidad no paró, aparecierón teóricos como Raymond Cattell, quien planteó el rasgo como elemento fundamental en su teoría, éste representa tendencias reactivas generales y nos indica características de conducta del individuo que son relativamente permanentes. El rasgo implica una configuración y regularidad de conducta a lo largo del tiempo y de las situaciones; Dollar y Miller, establecen una teoria fundamental en cuatro conceptos: los impulsos, las respuestas, los indicios y el refuerzo y Albert Bandura, quien planteó la teoría sobre el determinismo recíproco, que dice que el entorno de uno causa nuestro comportamiento pero, el comportamiento causa el ambiente, es decir, se causan mutuamente y postuló una interacción entre el ambiente, el comportamiento y los procesos psicológicos de la persona. Por mencionar solo algunos teóricos.

Teniendo en el presente una de las definiciones más completas, según la cual la personalidad representa un patrón profundamente incorporado y que muestra claramente rasgos cognitivos, afectivos y conductuales manifiestos que persisten por largos periodos de tiempo. Estos rasgos emergen de una complicada matriz de disposiciones biológicas y del aprendizaje experiencial. Este patrón es relativamente resistente a la extinción (Millón y Everly, 1994).

Encontrando de esta manera que, la personalidad se caracteriza por ser un patrón de rasgos, por su permanencia en el tiempo y por su resistencia al cambio, lo que podría remontarnos de alguna forma a la definición inicial.

Definición ampliada por Millón y Devis (2000), quienes la conciben como:

Un patrón complejo de características psicológicas profundamente arraigadas, que son en su mayor parte inconscientes y dificiles de cambiar, y se expresan automáticamente en casi todas las áreas del funcionamiento del individuo. Estos rasgos intrínsecos y generales surgen de una complicada matriz de determinantes biológicos y aprendizajes, y en última instancia comprenden el patrón idiosincrásico de percibir, sentir, pensar, afrontar y comportarse de un individuo (p. 4).

Para muchos autores la personalidad, puede entenderse mejor en términos de su contenido, debido a ello se han diseñado muchas taxonomías, es decir, la organización de las unidades básicas que la componen; encontrándose clasificaciones en torno a categorias, dimensiones y prototipos.

Este estudio utilizaró la clasificación en torno a dimensiones, puesto que los modelos dimensionales poseen varias ventajas, una de ellas sería la combinación de características clínicas o rasgos de personalidad, en un único perfil de fácil interpretación, haciendo una mirada global a los rasgos dentro de una dimensión y no a uno solo y asilado; otra ventaja es el favorecimiento de la representación individual y por último, la fuerza de los rasgos se mide cuantitativamente, así, cada característica se extiende en puntos de normalidad y anormalidad, dentro de un continuum, y no como fenómenos aislados (Millon y Davis, 2000). 
Dentro de este modelo, se ubica el modelo de los cinco factores (MCF) o Modelo Big Five (Modelo de los cinco grandes), que cuenta con representantes como Widiger, Costa y McCrae (Costa y McCrae, 1985; 1990; 1992 Costa y Widiger, 1993).

Este modelo es derivado directamente de estudios del léxico popular, de donde se codifican las palabras descriptivas, debido a su origen, se presentan algunos desacuerdo en cuanto a cómo nombrar cada uno de los cinco factores, aunque hay acuerdo en diferentes contextos y culturas en las siguientes denominaciones:

a) neuroticismo, inestabilidad emocional y susceptibilidad al malestar psicológico; b) extroversión, disposición a las interacciones interpersonales, a la actividad; c) apertura a la experiencia, aprecio por experimentar cosas nuevas, curiosidad; d) agradabilidad, buen carácter, altruismo; y e) responsabilidad, persistencia, ambición y control (Millón y Davis, 2000).

\section{Discusión y Conclusiones.}

Se podría unificar entonces algunos aspectos y factores que intervienen en la soledad, para definirla como una experiencia psicológica y emocional multidimensional y multicausada potencialmente estresante; es decir puede ser el resultado de carencias afectivas, sociales y/o físicas, reales o percibidas, que tienen un impacto diferencial sobre el funcionamiento de la salud física y psicológica del individuo. a) es multidimensional, porque involucra aspectos de personalidad (evaluaciones cognoscitivas, antecedentes de desarrollo afectivo y autoestima), de interacción social y de habilidades conductuales; b) es subjetiva y potencialmente estresante, ya que depende de la interpretación que haga el sujeto de la situación que está viviendo para que la carencia afectiva-social y/o fisica que identifique sea evaluada como una situación negativa, o bien, como una oportunidad de autoconocimiento; y c) es secuencial y multicausada, ya que puede estudiarse como un proceso que tiene antecedentes, manifestaciones y consecuencias, que varía en intensidad y duración a través de factores contribuyentes físico-situacionales, socio-culturales y de personalidad (Montero et al. 2001).

Dentro del marco referencial de la psicologia cognitiva, ha tenido un fuerte desarrollo la psicoterapia cognitiva de la depresión en cabeza de Aarón Beck en 1979, encontrándose la soledad como un factor de riesgo en depresión, lo que la ubica inevitablemente en la vía de la psicopatología, apareciendo fuertemente procesos como la atención y la percepción, estrategias de afrontamiento y solución de conflictos asociados a ella.

En esta misma línea se han estudiado los productos cognitivos, o pensamientos cognitivos que conducen al aislamiento, soledad y depresión. Y aparecen entonces teorias formuladas sobre la depresión, pero que involucran la soledad y el asilamiento, algunas de ellas son: la teoría de la desesperanza aprendida, la teoría reformulada de la desesperanza aprendida, la teoría de Beck, las teorías de la emoción y la teoría del attachment (Riso, 1992).

Se ha abordado el tema de la soledad, por un lado como el resultado de patrones disfuncionales de apego o vinculación con el otro, y además se ha planteado la soledad como el factor resultante de una interpretación errónea de nuestro entorno y de nuestras relaciones interpersonales. Encontrando conceptos como el dominio de desconexión y rechazo, descrito por Young (1990), como un área de la vida del individuo donde se posibilita 
la formación de esquemas maladaptativos tempranos de tipo, abandono o aislamiento; patrones de apego evitativos o ambivalentes; aislamiento social; que pueden confluir en rasgos marcados de la personalidad, o incluso en trastornos de personalidad cómo: personalidad limite, personalidad esquizoide, personalidad dependiente y personalidad evitativa.

Esto se evidencia cuando hay fallas en la afiliación que es la tendencia humana básica que nos lleva a buscar compañia como una manera de garantizar la supervivencia del individuo. De ahí que se generalice, que aquel que pueda prescindir de los demás es "esquizoide". Pues, todos necesitamos del contacto interpersonal. Por eso cuando se rompe la red de las relaciones sociales en las que estamos inmersos, podemos sentirnos extraños, distintos y vacios. La desconexión con el otro, nos desarraiga, nos quita la fuerza principal para seguir adelante. De hecho, hay evidencias que indican que una de las principales causas de la depresión infantily adulta es la ruptura afectiva con personas significativas (Riso, 2003).

La soledad emocional no se refiere tanto a la carencia de gente, sino a la desvinculación afectiva, el tema en cuestión sería la intimidad, el amor, el afecto de la pareja o de las amistades especiales. Podemos encontrarnos en medio de mucha gente, pero sentirnos emocionalmente solos. Pero no es lo mismo la soledad voluntaria a la soledad o aislamiento obligado, la primera puede ser una fuente de felicidad, autonomía, libertad, mientras que la segunda, altera la calidad de vida y la salud mental y fisica de un individuo (Riso, 2003).

Se encuentra además un acercamiento a la identificación de los factores que pueden causar la soledad: a) características de personalidad: introversión, timidez y ansiedad; b) baja autoestima: ("si creo que no valgo la pena, no me contacto con los demás"); c) déficit de habilidades sociales: es una incapacidad conductual o cognitiva, para poder establecer vínculos con los demás; d) características demográficas y e) estilos de apego: apego ambivalente y evitativo.

Mientras para mucha personas es bueno quedarse solas, para otras, sentirse solas puede convertirse en una experiencia dolorosa cuando sus relaciones sociales no son adecuadas, ni en calidad, ni en cantidad. Lo que denota también una diferencia entre momentos de soledad, que todos tenemos, y sentimiento de soledad (Valente y Aoyama, 1993).

Y después de este recorrido por la conceptualización de la soledad, de la percepción de soledad y de su vivencia por parte de la mujer, aparecen algunos factores de importancia significativa no solo en el contexto actual, el apoyo social real o percibido; la autoestima y la personalidad, como los que entrarían a mediar en esa percepción que se tenga de la soledad. Pero, estos factores no están interviniendo unos sobre otros, como simple causa y efecto (ER), están traspasando al sujeto mismo, en este caso, a la mujer que los vive. Mujer, que a su vez está inmersa en un mundo, en un sistema vincular particular y en un contexto históricocultural también particular y donde cada vez más de ellas viven solas o se encuentran solas, sin pareja, ellas han vivido la soledad, algunas la han sufrido, la han padecido, la han odiado, otras por el contrario la han degustado, la han disfrutado y hasta la han amado. Lo que podría indicar que es la forma como cada una de ellas se percibe frente a otro u otros y los percibe (ya sea por sus patrones vinculares, por su contexto histórico, cultural y geográfico), se podría entender entonces la soledad como un riesgo para su salud mental, o por el contrario podría ser un factor protector para la misma. 
Para finalizar surge entonces un interrogante: ¿Si la soledad de por sí no es la que causa el displacer, entonces qué hay en las mujeres que se encuentran solas, sin pareja, que hacen que unas la vivan como una oportunidad de intimidad, de autoprotección, reflexión e incluso de disfrute; mientras que otras la viven con dolor, con desesperanza, con temor e incluso con vergüenza? 


\section{Referencias Bibliográficas}

Alborch, C. (2001). Solas: gozos y sombras de una manera de vivir. Madrid: Temas de Hoy.

Aranda, C., Pando, M. y Aldrete, $\mathrm{M}^{\mathrm{a}}$ G. (2002). Jubilación, trastornos psicológicos y redes sociales de apoyo en el adulto mayor jubilado de Guadalajara, Jal. México. Revista de Psiquiatría de la Facultad de Medicina de Barcelona, 29, 169-174.

Barrón, A. (1996). Apoyo social. Aspectos teóricos y aplicaciones. España: Siglo Veintiuno.

Blascovich, J. y Tomaka, J. (1991). Measures of self-esteem. San Diego: Academic Press.

Borges, A., Pietro, P., Ricchetti, G., Hernandez, J.C. y Rodríguez, E. (2008). Validación Cruzada de la Factorización del Test UCLA de Soledad. Psicothema, 20 ( 4 ), 924- 927.

Borys, S. y Perlman, D. (1985). Gender differences in loneliness. Personality and Social Psychology Bulletin, 11, 63-74.

Caprara, G.V., Barbaranelli, C. y Borgogni, L. (1995). BFQ: Cuestionario "Big Five", Manual. España: TEA.

Castelló, J. (2005). Dependencia emocional: características y tratamiento. España: Alianza Editorial S.A.

Chen, L.J. y Chung, S.K. (2007). Loneliness, social connectedness, and family income among undergraduate females and males in Taiwan. Social Behavior and Personality, 35 (10), 1353-1364.

Cheng, H. y Furnham, A. (2002). Personality, peer relations, and self-confidence as predictors of happiness and loneliness. Journal of Adolescence, 25, 327-339

Cobb, S. (1976). Social Support as a moderator of life stress. Psychosomatic Medicine, $38,300-313$

Comrey, A.L. (1970). The Comrey Personality Scales. Son Diego: Ed. Plublishers. Comrey, A.L. (1973). A first course in factor analisys. Nueva York: Academic Press.

Comrey, A.L., y Lee, H.B. (1992). A first course in factor analisys, II ed. Hillsdale: Lawrences Erlbaum Associates.

Costa, P.T. y McCrae, R.R. (1992). The five-factor model of personality and its relevance to personality disorders. Journal of Personality Disorders, 6, 343-359.

Costa, P.T. y Widiger, T. (Eds.). (1993). Personality disorders and the five-factor model of personality. Washington, DC: American Psychological Association.

Epstein, S. (1979). The stability of behavior: On predicting most of the people much of the time. Journal of Personality and Social Psychology, 37, 1097-1126. 
Expósito, F. y Moya, M. (1999). Soledad y apoyo social. Revista de Psicología Social, 14 (2-3), 297-316.

Fierro, A. y Cardenal, V. (2001). Pertinencia de estilos de personalidad y variables cognitivas a indicadores de salud mental. Revista de Psicología General y Aplicada. 54 (2), 207226.

Gaviria, A., González, A., Palacio, C., García, J. y López, C. (2009). Factores asociados a la letalidad de intentos de suicidio en sujetos con trastorno depresivo mayor. Revista Colombiana de Psiquiatría, 4, 38(3), 446-463.

Gottlieb, B.H. (1983). Social support strategies: Guidelines for mental health practice. EE.UU.: Sage Publications.

Hogan, R. (1986). Hogan Personality Inventory Manual. Mineapolis: National Computer Systems.

Hojat, M. (1982). Loneliness as a function of selected personality variables. Journal of Clinical Psychology, 38 (1), 137- 141.

Hojat, M. y Crandall, R. (1989). Loneliness. Theory, Research, and Applications. Newbury Park: Sage.

Jong-Gierveld de J. (1987). Desarrollo y prueba de un modelo de soledad. Journal Personality Social Psychology, 53(1), 119-128.

Jong-Gierveld, de J. y Raadschelder, J. (1982). Types of loneliness. En: L.A. Peplau y D. Perlman. Loneliness. A Sourcebook of Current Theory, Research and Therapy (pp.105-119), Nueva York: Wiley y Sons.

Londoño, N.H., Castilla, J.F., Rogers, H.L., Palacio, J.E., Ochoa, N.L., Jaramillo, M.A. et al. (En prensa). Adaptación cultural y validación en Colombia del Cuestionario del estudio de desenlaces médicos de apoyo social (MOS-SSS).

Millon, T. y Everly Jr. G. (1994). La personalidad y sus trastornos. España: Ediciones Martinez Roca S.A.

Millon, T. y Davis, R. (2000). Trastornos de la personalidad: más allá del DSM-IV. España: Masson, S.A.

Montero, M., López, L. y Sánchez, J. (2001). La soledad como fenómeno Psicológico: Un análisis conceptual. Salud Mental, 24 (1), 19-27

Oviedo, M. y Cortés, R. (2007). Una Mirada a la Soledad. Colombia: Universidad Surcolombiana.

Peplau, L.A. y Perlman D. (1982). Loneliness. A Sourcebook of Current Theory, Research and Therapy. Nueva York: Wiley y Son. 
Riso, W. (1992). Depresión: avances recientes de la cognición y el procesamiento de la información. Colombia: Ediciones Gráficas Ltda.

Riso, W. (2003). Afirmando la Personalidad. Colombia: Panamericana Formas e Impresos S.A.

Rokach, A. (1988). Antecedentes de la Soledad: un análisis factorial. Journal Psychology, 123 (4), 39-54.

Rokach, A. (2005). Las causas de la soledad en jóvenes sin hogar. The Journal of Psychology, 139 (5), 469-480.

Rokach, A. y Neto, F. (2005). Edad, cultura, y los antecedentes de la soledad. Comportamiento Social y Personalidad, 33 (5), 477-494.

Rokach, A., Lechcier-kimiel y Safarov, A. (2006). La soledad de las personas con discapacidad fisica. Social Behavior and Personality, 34(6), 681-700.

Rokach, A., Matalon, R., Rokach, B. y Safarov, A. (2007). Los efectos de género y estado civil de la soledad de los ancianos. Social Behavior and Personality, 35(2),243-254.

Rosenberg, M. (1965). Society and the adolescent self-image. Princeton: Princeton University Press.

Russell, D., Peplau, L. y Ferguson M. (1978). Developing a measure of loneliness. J. Personality Assesment, 42, 290-294.

Russell, D., Peplau, D. y Cutrona, C. (1980). The revisited UCLA loneliness scale: Concurrent and discriminate validity evidence. J. Personality Social Psychology, 39, 472-480.

Russell, D., Cutrona, C., Rose, J. y Yuko, K. (1984). Social and emotional loneliness: and examination of Weiss's typology of loneliness. J. Personality Social Psychology, $46,1313-1321$.

Sadler, W.A. y Johnson T.B. (1980). From loneliness to anomia. Nueva York: International Universities Press.

Sherbourne, C. y Stewart, A. (1991). The MOS Social Support Survey. Social Scence and Medicine, 32, 705-14.

Uribe, D. (2008). Historia de las Civilizaciones. Colombia: Aguilar.

Valente, S. y Aoyama, D. (1993). Ayudar al paciente a vencer su soledad. Revista Nursing (Edición Española), 11(7), 26-28.

Weiss, R.S. (1973). La soledad: Una experiencia emocional y social deaislamiento. Cambridge: MIT Press. 
Weiss, R.S. (1982). Issues in the study of loneliness. En: L.A. Peplau y D. Perlman. Loneliness. A sourcebook of current theory, research and therapy (pp71-80). Nueva York: Wiley y Sons.

Wheeler, L., Reis, H. y Nezleck, J. (1983). Loneliness, social interaction, and sex roles. Journal of Personality and Social Psychology, 45, 943-953.

Wiseman, H., Mayseless, O. y Sharabany, R. (2006). Why are they lonely? Perceived quality of early relationships with parents, attachment, personality predispositions and loneliness in first-year university students. Personality and Individual Differences, 40, 237-248.

Young, J.E. (1982). Loneliness, depression and Cognitive Therapy: Theory and application. En: L.A. Peplau y D. Perlman. Loneliness. A sourcebook of current Theory, Research and Therapy (pp. 379- 405). Nueva York: Wiley y Sons.

Young, J. (1990). Cognitive therapy for personality disorders: a schema-focused approach. Florida: Professional resource exchange. Inc. 